\title{
Composition, Elastic Property and Packing Efficiency Predictions for Bulk Metallic Glasses in Binary, Ternary and Quaternary Systems
}

\author{
Carlos Ernesto Borja Soto *, Ignacio Alejandro Figueroa Vargas ${ }^{b}$, José Ramón Fonseca Velázquez, \\ Gabriel Ángel Lara Rodriguez ${ }^{b}$, Jorge Alejandro Verduzco Martínez ${ }^{a}$
}

\begin{abstract}
anstituto de Investigación en Metalurgia y Materiales, Universidad Michoacana de San Nicolás de Hidalgo - UMSNH, Fco. J. Mújica S/N, Col. Felícitas del Río, C.P. 58030, Morelia, Mich., México. ${ }^{b}$ Instituto de Investigaciones en Materiales, Universidad Nacional Autónoma de México - UNAM, Circuito Exterior S/N, Cd. Universitaria, C.P. 04510, México, D.F., México.
\end{abstract}

Received: September 8, 2015; Revised: November 25, 2015; Accepted: December 16, 2015

\begin{abstract}
The results of database based on the efficiency packed model for metallic glasses. The database contains the atomic radii information as well as elastic properties of the most commonly used alloying elements, permitting composition, packing efficiency and elastic property predictions to be made for binary, ternary and quaternary bulk metallic glasses. Twenty different alloys per system (binary, ternary and quaternary) experimentally reported in the literature were compared with those estimated by the database. Comparison charts and diagrams showed good agreement between the composition predictions and those reported from the experimentally processed metallic glasses. The elastic properties predictions could be used to elaborate Blackman diagrams in order to know, in advance, the intrinsic toughness that the investigated alloys might present. The database is intended for designing bulk metallic glasses. Finally, some quaternary alloys were experimentally produced based on the prediction obtained with the database, showing a glassy phase. The microhardness values obtained experimentally of the $\mathrm{Zr}_{57.52} \mathrm{Ag}_{10.62} \mathrm{Al}_{10.62} \mathrm{Co}_{21.24}, \mathrm{Zr}_{57.19} \mathrm{Al}_{10.7} \mathrm{Ni}_{10.7} \mathrm{Cu}_{21.41}$ and $\mathrm{Hf}_{60.22} \mathrm{Al}_{9.95} \mathrm{Cu}_{9.95} \mathrm{Ni}_{19.89}$ alloys, are 3.8, 4.0 and $3.6 \mathrm{GPa}$, respectively. The Young's modulus calculated using microhardness values $\left(\mathrm{E} / \mathrm{H}_{\mathrm{v}}=20\right)$ are closed to the values obtained by the "mixing rules".
\end{abstract}

Keywords: Bulk metallic glasses, Packing efficiency, Glass formation

\section{Introduction}

Despite new metallic glass systems being continuously investigated and reported, and a broadening of the number of engineering applications for this kind of material, the discovery, development and manufacture of bulk metallic glass (BMG) systems is still a complex and long process. Materials science and engineering states that when a new material is to be produced, prior to this, some properties would be expected from that new material, from its shape, component materials, structure, etc. The other way around would be, knowing the structure, component materials and production of new materials in order to fit some specification. However, for metallic glasses, the structure is still an unsolved puzzle, even though the international community has witnessed their advantageous properties and behaviour i.e. high strength, corrosion and wear resistant, chemical inertness, high toughness, among others.

In 1926, Goldschmidt ${ }^{1}$ correlated the ability to form a glass with the value of the radius ratio $\mathrm{r}_{\mathrm{A}} / \mathrm{r}_{\mathrm{O}}$ for oxides $\mathrm{A}_{\mathrm{m}} \mathrm{O}_{n}$. He found that for all the oxides which had been prepared in the vitreous form, the radius ratio was around $0.2-0.4$. Zachariasen ${ }^{2}$, proposed a model where the $\mathrm{SiO}_{4}$ polyhedron is repeated to produce a continuous random network (CRN). Regarding the study of BMG structure, different structural

*e-mail: borjas.ce@gmail.com models have been proposed in the past 50 years; Bernal's "dense random packing"3 model and Miracle's "efficient solute-centred cluster packing", amongst many others. The results of the latter are consistent with the high densities measured in bulk metallic glasses. Table 1 summaries some historical ideas pertaining to atomic packing and glass formation.

Packing in BMG is very dense with melt viscosities that are several orders of magnitude higher than in pure metallic melts. The dense packing accomplished by structural and chemical atomic ordering below the glass transition temperature also brings the BMG-forming liquids energetically and entropically closer to the corresponding crystalline state. These factors lead to slow crystallisation kinetics and consequentially to high glass forming ability $(G F A)^{12}$. Since GFA is an influential factor in studying the formation of BMG, the search for systems with sufficiently high GFA is a critical task in this field.

The mechanical behaviour of metallic glasses is also one of the most important topics, attracting a huge amount of research effort. Given its large practical relevance to the implementation of BMG as structural materials in real applications, it is important to know whether a material shows plastic deformation or brittle rupture under certain loading conditions. As a tool for this, the use of Blackman diagrams 
Table 1. Summarised historical events of atomic packing and glass formation.

\begin{tabular}{|c|c|c|c|}
\hline Who & When & What & Related to \\
\hline Goldschmidt $^{1}$ & 1926 & $\begin{array}{l}\text { Tetrahedral configurations were necessary for the formation of } \\
\text { a glass }\end{array}$ & Oxides of the type $\mathrm{A}_{\mathrm{m}} \mathrm{O}_{\mathrm{n}}$ \\
\hline Zachariasen $^{2}$ & 1932 & Continuous random network (CRN) & $\mathrm{SiO}_{4}$ \\
\hline Sun $^{5}$ & 1946 & $\begin{array}{l}\text { Model to obtain long range or network of atoms to form a glassy } \\
\text { phase }\end{array}$ & Oxides of the type M-O single bound \\
\hline Bernal, Mason $^{3}$ & 1959 & Model of dense random packing (DRP) or rigid spheres & Bernal holes \\
\hline Polk $^{6}$ & 1972 & Model to describe transition metal - metalloid glassy systems & Metallic glasses \\
\hline $\begin{array}{l}\text { Egami } \\
\text { Waseda }^{7}\end{array}$ & 1984 & Relation between glass formability and atomic size mismatch & Binary metallic glassy alloys \\
\hline Granato $^{8}$ & 1993 & $\begin{array}{l}\text { The main roles of interstitial atoms is in destabilising the } \\
\text { crystalline phase }\end{array}$ & Frozen glassy and liquid states alloys \\
\hline Egami $^{9}$ & 1997 & Topological instability applied to local atomic structure & Metallic elements and alloys \\
\hline Senkov, Miracle ${ }^{10}$ & 2001 & Interstitial model for glass formation & Prediction of glass formation \\
\hline Egami $^{11}$ & 2003 & $\begin{array}{l}\text { Identified several conditions that would favour multicomponent } \\
\text { bulk metallic glass formation }\end{array}$ & Prediction of BMG-formation \\
\hline Miracle $^{4}$ & 2004 & $\begin{array}{l}\text { Model to determine the alloy constituent concentration based on } \\
\text { a topological atomistic approach }\end{array}$ & $\begin{array}{l}\text { Dense packing of atomic clusters for } \\
\text { glass formation }\end{array}$ \\
\hline
\end{tabular}

have been suggested and employed ${ }^{13}$ to explain the tendency for permanent deformation that a system may exhibit.

The work presented in this manuscript is based on several theoretical models for metallic glasses in terms of glass formation and elastic properties, and is intended to offer an alternative route to design and obtain BMG by estimating the system with GFA from a structural perspective (densest atomic packing) and predict its theoretical elemental elastic properties, from which some indication of plasticity can be obtained. In addition to the above, quaternary alloys were experimentally produced in order to get bulk metallic glasses.

\section{Experimental and theoretical calculations}

\subsection{Theoretical chemical composition calculation}

Chemical compositions were calculated based on a sphere-packing scheme (solute-centred clusters occupying an f.c.c. cluster unit cell $)^{4}$. The Miracle's model includes the calculation of the three-dimensional coordination number $\mathrm{N}^{\mathrm{T}}$, which is obtained for a radius ratio $\mathrm{R}^{*}$ for maximum packing efficiency ${ }^{14}$. The efficiency packing was calculated from the chemical composition ${ }^{14}$ and cluster unit cell length ${ }^{15}$.

\subsection{Theoretical elastic properties calculation}

The elastic modulus predictions were also carried out for different glassy alloys systems taken from the literature. The estimations were made by applying the "rule of mixtures" approach ${ }^{16-17}$ once having estimated the composition for the alloy using the efficient cluster-packing model. In the same way as before, theoretical estimations were compared to experimental values reported in the open literature. Twenty different values for Poisson's ratio for typical BMG were taken from ${ }^{17}$; for these twenty systems elastic constants $c_{11}, c_{12}$ and $c_{44}$ were also predicted in order to elaborate a Blackman diagram (plotting $c_{12} / c_{11}$ vs. $c_{44} / c_{11}$ ). The elastic constants $c_{\mathrm{ij}}$ were calculated with 1 - 3 equations ${ }^{17-18}$.

$c_{11}=K+(4 / 3) G$ $c_{12}=\left(3 K-c_{11}\right) / 2$

$c_{44}=G$

The transition between brittle and tough regimes is at $v_{\text {crit }}=0.31-0.32^{19}$. Higher values of $v$ give higher fracture energy ${ }^{19}$. In other words, the larger the $v$ is, the more ductile the BMG become, and small variation of $v$ will significantly change the ductility ${ }^{17}$. Several theoretical compositions obtained from the database were compared to those reported in the literature, and twenty different alloys per system (binary, ternary and quaternary), reported in the literature, are considered here.

\subsection{Experimental method}

Alloys with nominal compositions $\mathrm{Zr}_{57.52} \mathrm{Ag}_{10.62} \mathrm{Al}_{10.62} \mathrm{Co}_{21.24}$, $\mathrm{Zr}_{57.19} \mathrm{Al}_{10.7} \mathrm{Ni}_{10.7} \mathrm{Cu}_{21.41}$, and $\mathrm{Hf}_{60.22} \mathrm{Al}_{9.95} \mathrm{Cu}_{9.95} \mathrm{Ni}_{19.89}$ were prepared with pure elements (purity $>99.99 \%$ ). The alloys were prepared under Ti-gettered inert atmosphere of argon (high purity $>99.9$ ) using the arc-melting technique. All the ingots were re-melted five times in order to achieve a chemical homogeneity. Then, the molten alloys were poured into a copper mold with an internal cylindrical cavity of $2 \mathrm{~mm}$ diameter $\times 12 \mathrm{~mm}$ length by suction casting technique. The structure of the samples was characterized by means of X-ray diffraction with a Siemens D5000 diffractometer, using $\mathrm{Cu} \mathrm{K}$ radiation. Finally, the hardness of the samples was carried out with a HMV-G21D hardness tester, in order to calculate the Young's modulus values.

\section{Results and discussion}

\subsection{Chemical composition}

The prediction for the binary $\mathrm{Pd}-\mathrm{Si}$, referred to in Table 2 as alloy 1 , being the compositions $\mathrm{Pd}_{82} \mathrm{Si}_{18}$ and $\mathrm{Pd}_{82.96} \mathrm{Si}_{17.04}$, which are the reported and predicted, respectively, showed very good agreement. Other examples are the binaries $\mathrm{Co}-\mathrm{B}, \mathrm{Al}-\mathrm{Cu}, \mathrm{Co}-\mathrm{Zr}, \mathrm{Au}-\mathrm{Si}$, where the difference between 
the reported and predicted composition was small. From the twenty binary alloys investigated, about five showed a significant difference between the reported and predicted compositions. Composition predictions for the twenty different binary alloys were obtained; these alloys are listed in Table 2. It can be observed that the calculated packaging efficiency for the majority of the alloys was approximately $40 \%$. The packing efficiency for $\mathrm{Co}_{77.61} \mathrm{~B}_{22.39}, \mathrm{Ba}_{77.7} \mathrm{Al}_{22.3}$, $\mathrm{Ba}_{77.09} \mathrm{Zn}_{22.91}, \mathrm{Ba}_{77.09} \mathrm{Zn}_{22.91}$ and $\mathrm{Fe}_{77.79} \mathrm{~B}_{22.21}$ binary alloys was about $50 \%$. Figure 1 shows the absolute difference between calculated and reported compositions alloys. The absolute difference of chemical composition for most alloys compared is less than $14 \%$. The $\mathrm{Ni}-\mathrm{Nb}\left(\mathrm{Ni}_{59.5} \mathrm{Nb}_{40.5}, \mathrm{Ni}_{89.69} \mathrm{Nb}_{10.31}\right.$, $\mathrm{Cu}_{64.5} \mathrm{Zr}_{35.5}$ and $\left.\mathrm{Cu}_{90.94} \mathrm{Zr}_{9.06}\right)$ binary system, showed $30 \%$ of difference, approximately.

Table 3 shows 20 ternary metallic glass compositions reported, it also includes the compositions and packing efficiency calculated with the database. The 4, 5, 7, 11, 15 , and 20 alloy systems showed good agreement between calculated and reported ternary compositions as shown in Figure 2. However, for the $\mathrm{Cu}-\mathrm{Zr}-\mathrm{Al}, \mathrm{Mg}-\mathrm{Gd}-\mathrm{Cu}$ and $\mathrm{Cu}-\mathrm{Zr}$-Be ternary alloy systems a significant difference was evident. On the other hand, the packing efficiency calculated for the $\mathrm{Fe}_{75.76} \mathrm{Y}_{6.06} \mathrm{~B}_{18.18}$ alloy was exceptional, i.e. 96.41\%. In addition, the difference between the calculated and reported composition was very small.

Table 4 shows the quaternary compositions of BMG reported and calculated. Figure 3 shows good agreement of quaternary compositions. The chemical compositions difference of the alloys reported and calculated was less than $10 \%$ for most of the alloy systems studied.

\subsection{Elastic properties}

The elastic modulus (E) and shear modulus (G) estimations for twenty different alloys were also calculated and compared with those reported in the literature. The corresponding alloys are listed in Tables 5 and 6, respectively and plotted in Figure 4. (It was possible to estimate values for bulk modulus (K), too, but these are not included in this work).

Regarding the Young's modulus, E, the $\mathrm{Mg}_{65} \mathrm{Cu}_{25} \mathrm{~Tb}_{10}$ system reports $51.3 \mathrm{GPa}$ while its predicted value was $54.8 \mathrm{GPa}$; the $\mathrm{Hf}_{55} \mathrm{Ni}_{25} \mathrm{Al}_{20}$ reports $117.63 \mathrm{GPa}^{13}$ and the predicted value is $113.8 \mathrm{GPa}\left(\mathrm{Hf}_{60} \mathrm{Ni}_{30} \mathrm{Al}_{10}\right)$. In general, the similarity between the reported and predicted elastic

Table 2. Predicted and reported alloy compositions for binary systems ${ }^{20-33}$.

\begin{tabular}{|c|c|c|c|c|c|c|c|}
\hline $\mathrm{N}^{\circ}$. alloy & $\begin{array}{c}\text { Reported } \\
\text { composition }\end{array}$ & $\begin{array}{l}\text { Predicted } \\
\text { composition }\end{array}$ & \%Pack. Eff. & $\mathbf{N}^{\circ}$. alloy & $\begin{array}{c}\text { Reported } \\
\text { Composition }\end{array}$ & $\begin{array}{c}\text { Predicted } \\
\text { Composition }\end{array}$ & \%Pack. Eff. \\
\hline 1 & $\mathrm{Pd}_{82} \mathrm{Si}_{18}$ & $\mathrm{Pd}_{82.96} \mathrm{Si}_{17.04}$ & 44.76 & 11 & $\mathrm{Al}_{91} \mathrm{Er}_{9}$ & $\mathrm{Al}_{91.22} \mathrm{Er}_{8.78}$ & 41.38 \\
\hline 2 & $\mathrm{Pd}_{81} \mathrm{Si}_{19}$ & $\mathrm{Pd}_{82.96} \mathrm{Si}_{17.04}$ & 44.76 & 12 & $\mathrm{Au}_{81.4} \mathrm{Si}_{18.6}$ & $\mathrm{Au}_{81.98} \mathrm{Si}_{18.02}$ & 41.69 \\
\hline 3 & $\mathrm{Pd}_{80} \mathrm{Si}_{20}$ & $\mathrm{Pd}_{82.96} \mathrm{Si}_{17.04}$ & 44.76 & 13 & $\mathrm{Au}_{83} \mathrm{Si}_{17}$ & $\mathrm{Au}_{81.98} \mathrm{Si}_{18.02}$ & 41.69 \\
\hline 4 & $\mathrm{Ca}_{66.4} \mathrm{Al}_{33.6}$ & $\mathrm{Ca}_{79.86} \mathrm{Al}_{20.14}$ & 44.76 & 14 & $\mathrm{Au}_{80} \mathrm{Si}_{20}$ & $\mathrm{Au}_{81.98} \mathrm{Si}_{18.02}$ & 41.69 \\
\hline 5 & $\mathrm{Ni}_{59.5} \mathrm{Nb}_{40.5}$ & $\mathrm{Ni}_{89.69} \mathrm{Nb}_{10.31}$ & 45.75 & 15 & $\mathrm{Au}_{75} \mathrm{Si}_{25}$ & $\mathrm{Au}_{81.98} \mathrm{Si}_{18.02}$ & 41.69 \\
\hline 6 & $\mathrm{Cu}_{64.5} \mathrm{Zr}_{35.5}$ & $\mathrm{Cu}_{90.94} \mathrm{Zr}_{9.06}$ & 42.22 & 16 & $\mathrm{Ba}_{72} \mathrm{Al}_{28}$ & $\mathrm{Ba}_{77.7} \mathrm{Al}_{22.3}$ & 51.85 \\
\hline 7 & $\mathrm{Cu}_{90} \mathrm{Zr}_{10}$ & $\mathrm{Cu}_{90.94} \mathrm{Zr}_{9.06}$ & 42.22 & 17 & $\mathrm{Ba}_{76} \mathrm{Ga}_{24}$ & $\mathrm{Ba}_{77.05} \mathrm{Ga}_{22.95}$ & 50.37 \\
\hline 8 & $\mathrm{Co}_{77} \mathrm{~B}_{23}$ & $\mathrm{Co}_{77.61} \mathrm{~B}_{22.39}$ & 51.65 & 18 & $\mathrm{Ba}_{76} \mathrm{Zn}_{24}$ & $\mathrm{Ba}_{77.09} \mathrm{Zn}_{22.91}$ & 50.46 \\
\hline 9 & $\mathrm{Al}_{82.7} \mathrm{Cu}_{17.3}$ & $\mathrm{Al}_{84.22} \mathrm{Cu}_{15.78}$ & 41.75 & 19 & $\mathrm{Ca}_{87.5} \mathrm{Ag}_{12.5}$ & $\mathrm{Ca}_{80.06} \mathrm{Ag}_{19.94}$ & 46.17 \\
\hline 10 & $\mathrm{Co}_{91} \mathrm{Zr}_{9}$ & $\mathrm{Co}_{91.22} \mathrm{Zr}_{8.78}$ & 41.14 & 20 & $\mathrm{Fe}_{78} \mathrm{~B}_{22}$ & $\mathrm{Fe}_{77.79} \mathrm{~B}_{22.21}$ & 52.07 \\
\hline
\end{tabular}

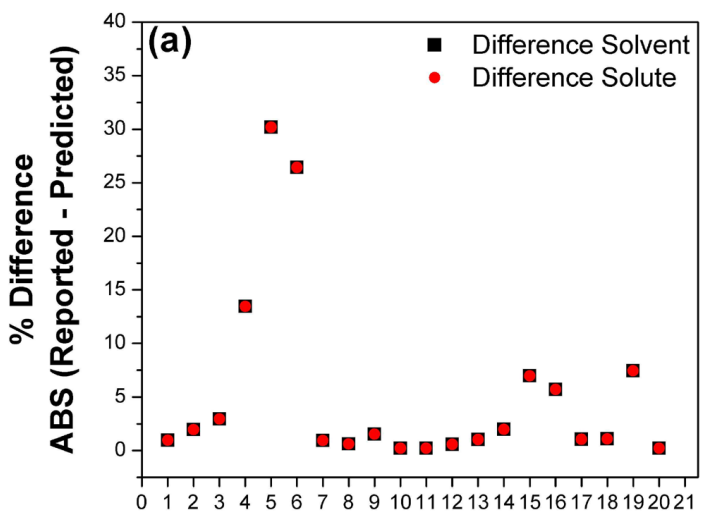

No. of Alloy

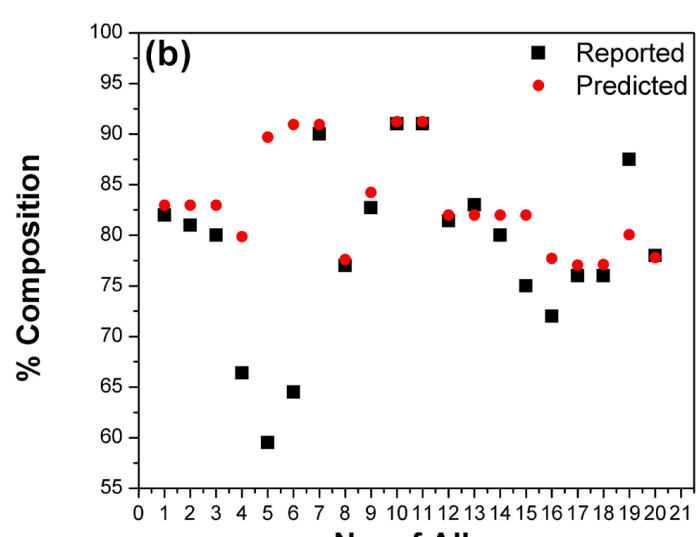

No. of Alloy

Figure 1. Difference of chemical compositions of binary alloys. (a) Absolute difference of composition predictions for twenty different binary alloys and (b) Difference of solvents predictions for twenty different binary alloys. 
Table 3. Predicted and reported alloy compositions for ternary systems ${ }^{34-53}$.

\begin{tabular}{cccccccc}
\hline $\begin{array}{c}\text { No. } \\
\text { alloy }\end{array}$ & $\begin{array}{c}\text { Reported } \\
\text { composition }\end{array}$ & $\begin{array}{c}\text { Predicted } \\
\text { composition }\end{array}$ & \%Pack. Eff. & No. alloy & $\begin{array}{c}\text { Reported } \\
\text { composition }\end{array}$ & $\begin{array}{c}\text { Predicted } \\
\text { composition }\end{array}$ & $\begin{array}{c}\text { \%Pack. } \\
\text { Eff. }\end{array}$ \\
\hline 1 & $\mathrm{Gd}_{55} \mathrm{Al}_{20} \mathrm{Co}_{25}$ & $\mathrm{Gd}_{60.24} \mathrm{Al}_{13.25} \mathrm{Co}_{26.51}$ & 65.64 & 11 & $\mathrm{Sm}_{60} \mathrm{Al}_{10} \mathrm{Fe}_{30}$ & $\mathrm{Sm}_{60.24} \mathrm{Al}_{13.25} \mathrm{Fe}_{26.51}$ & 65.64 \\
2 & $\mathrm{Cu}_{46} \mathrm{Zr}_{46} \mathrm{Al}_{8}$ & $\mathrm{Cu}_{76.86} \mathrm{Zr}_{7.71} \mathrm{Al}_{15.43}$ & 60.64 & 12 & $\mathrm{Gd}_{56} \mathrm{Al}_{24} \mathrm{Co}_{20}$ & $\mathrm{Gd}_{60.24} \mathrm{Al}_{13.25} \mathrm{Co}_{26.51}$ & 65.64 \\
3 & $\mathrm{Mg}_{65} \mathrm{Gd}_{25} \mathrm{Cu}_{10}$ & $\mathrm{Mg}_{73.53} \mathrm{Gd}_{8.11} \mathrm{Cu}_{24.32}$ & 59.28 & 13 & $\mathrm{Gd}_{55} \mathrm{Al}_{25} \mathrm{Ni}_{20}$ & $\mathrm{Gd}_{60.24} \mathrm{Al}_{13.25} \mathrm{Ni}_{26.51}$ & 65.64 \\
4 & $\mathrm{Mg}_{75} \mathrm{Gd}_{10} \mathrm{Ni}_{15}$ & $\mathrm{Mg}_{73.53} \mathrm{Gd}_{8.82} \mathrm{Ni}_{17.65}$ & 71.81 & 14 & $\mathrm{Nd}_{60} \mathrm{Al}_{10} \mathrm{Co}_{30}$ & $\mathrm{Nd}_{56.81} \mathrm{Al}_{10.8} \mathrm{Co}_{32.39}$ & 45.7 \\
5 & $\mathrm{Zr}_{58} \mathrm{Al}_{12} \mathrm{Co}_{30}$ & $\mathrm{Zr}_{56.81} \mathrm{Al}_{10.8} \mathrm{Co}_{32.39}$ & 45.7 & 15 & $\mathrm{Ce}_{70} \mathrm{Al}_{10} \mathrm{Cu}_{20}$ & $\mathrm{Ce}_{69.44} \mathrm{Al}_{15.28} \mathrm{Cu}_{15.28}$ & 61.57 \\
6 & $\mathrm{Cu}_{47.5} \mathrm{Zr}_{40} \mathrm{Be}_{12.5}$ & $\mathrm{Cu}_{76.86} \mathrm{Zr}_{7.71} \mathrm{Be}_{15.43}$ & 69.74 & 16 & $\mathrm{Pr}_{60} \mathrm{Al}_{10} \mathrm{Fe}_{10}$ & $\mathrm{Pr}_{63.68} \mathrm{Al}_{12.11} \mathrm{Fe}_{24.21}$ & 67.15 \\
7 & $\mathrm{Zr}_{58} \mathrm{Al}_{12} \mathrm{Cu}_{30}$ & $\mathrm{Zr}_{56.81} \mathrm{Al}_{10.8} \mathrm{Cu}_{32.39}$ & 45.7 & 17 & $\mathrm{~Tb}_{65} \mathrm{Al}_{10} \mathrm{Fe}_{25}$ & $\mathrm{~Tb}_{60.24} \mathrm{Al}_{13.25} \mathrm{Fe}_{26.51}$ & 60.59 \\
8 & $\mathrm{Hf}_{62} \mathrm{Al}_{13} \mathrm{Ni}_{15}$ & $\mathrm{Hf}_{66.67} \mathrm{Al}_{11.11} \mathrm{Ni}_{22.22}$ & 67.22 & 18 & $\mathrm{Y}_{56} \mathrm{Al}_{24} \mathrm{Co}_{20}$ & $\mathrm{Y}_{60.24} \mathrm{Al}_{13.25} \mathrm{Co}_{26.52}$ & 65.64 \\
9 & $\mathrm{Fe}_{72} \mathrm{Y}_{6} \mathrm{~B}_{22}$ & $\mathrm{Fe}_{75.76} \mathrm{Y}_{6.06} \mathrm{~B}_{18.18}$ & 96.41 & 19 & $\mathrm{Fe}_{80} \mathrm{P}_{11} \mathrm{C}_{9}$ & $\mathrm{Fe}_{72.46} \mathrm{P}_{13.77} \mathrm{C}_{13.77}$ & 37.79 \\
10 & $\mathrm{Sm}_{55} \mathrm{Al}_{25} \mathrm{Co}_{20}$ & $\mathrm{Sm}_{60.24} \mathrm{Al}_{13.25} \mathrm{Co}_{26.51}$ & 65.64 & 20 & $\mathrm{La}_{62} \mathrm{Al}_{14} \mathrm{Cu}_{24}$ & $\mathrm{La}_{60.24} \mathrm{Al}_{13.25} \mathrm{Cu}_{26.51}$ & 65.64 \\
\hline
\end{tabular}
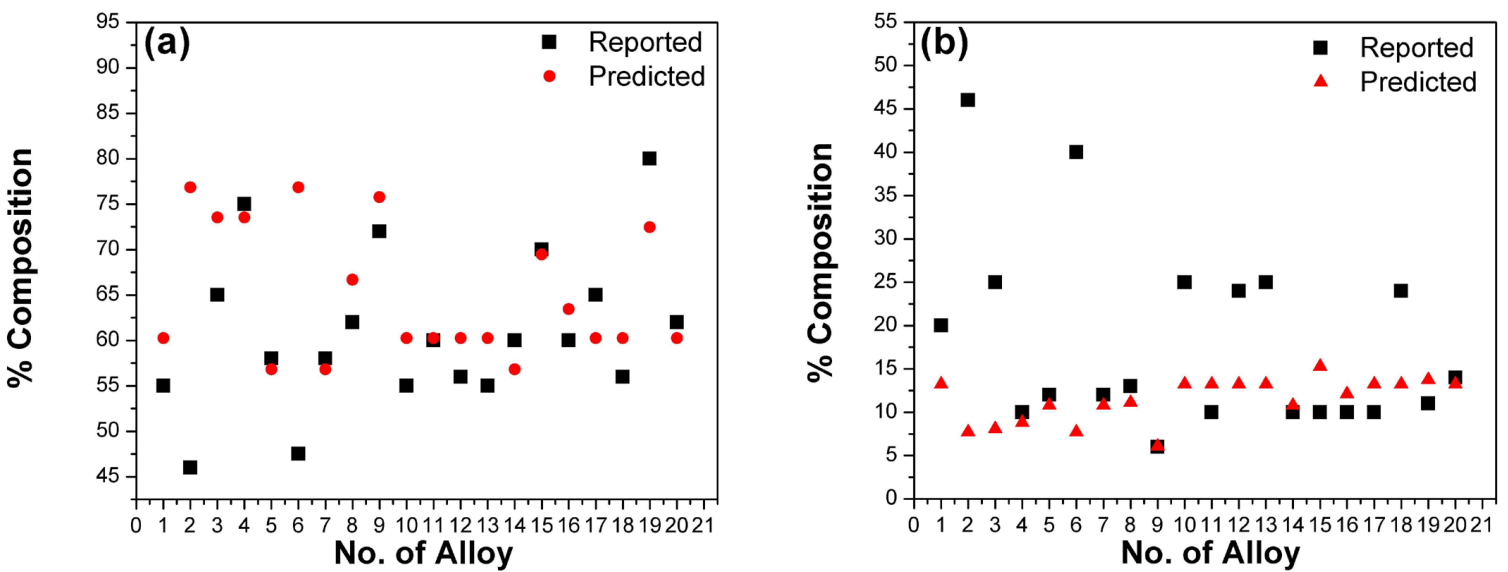

Figure 2. Difference of chemical compositions of ternary alloys. (a) Composition predictions of solvents for twenty different ternary alloys and (b) Difference of ternary large solutes for the predicted and calculated alloys.

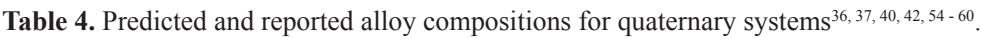

\begin{tabular}{|c|c|c|c|c|c|c|c|}
\hline $\begin{array}{l}N^{\circ} \text {. } \\
\text { alloy }\end{array}$ & $\begin{array}{c}\text { Reported } \\
\text { composition }\end{array}$ & Predicted composition & $\begin{array}{c}\text { \%Pack. } \\
\text { Eff. }\end{array}$ & $\begin{array}{l}\mathrm{N}^{\circ} \text {. } \\
\text { alloy }\end{array}$ & $\begin{array}{c}\text { Reported } \\
\text { Composition }\end{array}$ & Predicted Composition & $\begin{array}{c}\text { \%Pack. } \\
\text { Eff. }\end{array}$ \\
\hline 1 & $\mathrm{Pt}_{57.5} \mathrm{Cu}_{14.7} \mathrm{Ni}_{5.3} \mathrm{P}_{22.5}$ & $\mathrm{Pt}_{60.76} \mathrm{Cu}_{9.81} \mathrm{Ni}_{9.81} \mathrm{P}_{19.62}$ & 50.23 & 11 & $\mathrm{Zr}_{53} \mathrm{Ag}_{9} \mathrm{Al}_{23.5} \mathrm{Co}_{14.5}$ & $\mathrm{Zr}_{57.52} \mathrm{Ag}_{10.6} \mathrm{Al}_{10.62} \mathrm{Co}_{21.24}$ & 45.37 \\
\hline 2 & $\mathrm{Ce}_{68} \mathrm{Al}_{10} \mathrm{Nb}_{2} \mathrm{Cu}_{20}$ & $\mathrm{Ce}_{52.56} \mathrm{Al}_{11.86} \mathrm{Nb}_{11.86} \mathrm{Cu}_{23.72}$ & 46.64 & 12 & $\mathrm{Gd}_{55} \mathrm{Al}_{20} \mathrm{Mn}_{3} \mathrm{Ni}_{22}$ & $\mathrm{Gd}_{53.01} \mathrm{Al}_{11.75} \mathrm{Mn}_{11.75} \mathrm{Ni}_{23.49}$ & 48.27 \\
\hline 3 & $\mathrm{Mg}_{58.5} \mathrm{Y}_{2} \mathrm{Gd}_{9} \mathrm{Cu}_{30.5}$ & $\mathrm{Mg}_{67.83} \mathrm{Y}_{8.04} \mathrm{Gd}_{8.04} \mathrm{Cu}_{16.09}$ & 52.92 & 13 & $\mathrm{Zr}_{61} \mathrm{Ti}_{2} \mathrm{Al}_{12} \mathrm{Cu}_{25}$ & $\mathrm{Zr}_{60.39} \mathrm{Ti}_{9.9} \mathrm{Al}_{9.9} \mathrm{Cu}_{19.8}$ & 48.39 \\
\hline 4 & $\mathrm{Mg}_{74} \mathrm{Gd}_{10} \mathrm{Ag}_{1} \mathrm{Ni}_{15}$ & $\mathrm{Mg}_{67.83} \mathrm{Gd}_{8.04} \mathrm{Ag}_{8.04} \mathrm{Ni}_{16.09}$ & 58.13 & 14 & $\mathrm{Ni}_{60} \mathrm{Pd}_{20} \mathrm{P}_{17} \mathrm{~B}_{3}$ & $\mathrm{Ni}_{67.18} \mathrm{Pd}_{8.2} \mathrm{P}_{8.2} \mathrm{~B}_{16.41}$ & 66.95 \\
\hline 5 & $\mathrm{Mg}_{73} \mathrm{Gd}_{10} \mathrm{Ag}_{2} \mathrm{Ni}_{15}$ & $\mathrm{Mg}_{67.83} \mathrm{Gd}_{8.04} \mathrm{Ag}_{8.04} \mathrm{Ni}_{16.09}$ & 58.13 & 15 & $\mathrm{Fe}_{67.7} \mathrm{Dy}_{6} \mathrm{Mo}_{3.76} \mathrm{~B}_{22.6}$ & $\mathrm{Fe}_{75.68} \mathrm{Dy}_{6.08} \mathrm{Mo}_{6.08} \mathrm{~B}_{12.16}$ & 61.51 \\
\hline 6 & $\mathrm{Mg}_{71} \mathrm{Gd}_{10} \mathrm{Ag}_{4} \mathrm{Ni}_{17}$ & $\mathrm{Mg}_{67.83} \mathrm{Gd}_{8.04} \mathrm{Ag}_{8.04} \mathrm{Ni}_{16.09}$ & 58.13 & 16 & $\mathrm{Fe}_{68} \mathrm{Y}_{6} \mathrm{~W}_{4} \mathrm{~B}_{22}$ & $\mathrm{Fe}_{76.15} \mathrm{Y}_{5.96} \mathrm{~W}_{5.96} \mathrm{~B}_{11.92}$ & 61.78 \\
\hline 7 & $\mathrm{Mg}_{70} \mathrm{Gd}_{10} \mathrm{Ag}_{5} \mathrm{Ni}_{15}$ & $\mathrm{Mg}_{67.83} \mathrm{Gd}_{8.04} \mathrm{Ag}_{8.04} \mathrm{Ni}_{16.09}$ & 58.13 & 17 & $\mathrm{Zr}_{46} \mathrm{Ag}_{8.4} \mathrm{Al}_{8} \mathrm{Cu}_{37.6}$ & $\mathrm{Zr}_{57.52} \mathrm{Ag}_{10.62} \mathrm{Al}_{10.62} \mathrm{Cu}_{21.24}$ & 44.7 \\
\hline 8 & $\mathrm{Mg}_{69} \mathrm{Gd}_{10} \mathrm{Ag}_{6} \mathrm{Ni}_{15}$ & $\mathrm{Mg}_{67.83} \mathrm{Gd}_{8.04} \mathrm{Ag}_{8.04} \mathrm{Ni}_{16.09}$ & 58.13 & 18 & $\mathrm{Fe}_{68} \mathrm{Y}_{6} \mathrm{Mo}_{4} \mathrm{~B}_{22}$ & $\mathrm{Fe}_{76.15} \mathrm{Y}_{5.96} \mathrm{Mo}_{5.96} \mathrm{~B}_{11.92}$ & 61.94 \\
\hline 9 & $\mathrm{Mg}_{68} \mathrm{Gd}_{10} \mathrm{Ag}_{7} \mathrm{Ni}_{15}$ & $\mathrm{Mg}_{67.83} \mathrm{Gd}_{8.04} \mathrm{Ag}_{8.04} \mathrm{Ni}_{16.09}$ & 58.13 & 19 & $\mathrm{Fe}_{70} \mathrm{Y}_{6} \mathrm{Ni}_{2} \mathrm{~B}_{22}$ & $\mathrm{Fe}_{76.15} \mathrm{Y}_{5.96} \mathrm{Ni}_{5.96} \mathrm{~B}_{11.92}$ & 66.48 \\
\hline 10 & $\mathrm{Zr}_{53} \mathrm{Ag}_{5} \mathrm{Al}_{23.5} \mathrm{Co}_{18.5}$ & $\mathrm{Zr}_{57.52} \mathrm{Ag}_{10.62} \mathrm{Al}_{10.62} \mathrm{Co}_{21.24}$ & 45.37 & 20 & $\mathrm{Fe}_{66} \mathrm{Y}_{6} \mathrm{Co}_{6} \mathrm{~B}_{22}$ & $\mathrm{Fe}_{76.15} \mathrm{Y}_{5.96} \mathrm{Co}_{5.96} \mathrm{~B}_{11.92}$ & 66.27 \\
\hline
\end{tabular}

moduli is rather good. However, it can be noticed that some of the composition predictions, even though they did show a slight deviation from those reported in literature, kept a narrow correlation with regards to the elastic modulus values. The elastic property predictions also showed good correlation with the corresponding experimentally reported data (Tables 5, 6 and Figure 4). The $\mathrm{Mg}_{65} \mathrm{Cu}_{25} \mathrm{~Tb}_{10}$ ternary system reports a shear modulus, $\mathrm{G}$, of $19.6 \mathrm{GPa}^{13}$ and its corresponding prediction gives $20.8 \mathrm{GPa}\left(\mathrm{Mg}_{79.03} \mathrm{Cu}_{10.48} \mathrm{~Tb}_{10.48}\right)$; the quaternary $\mathrm{Gd}_{40} \mathrm{Y}_{16} \mathrm{Al}_{24} \mathrm{Co}_{20}$ reports a $\mathrm{G}$ of $23.5 \mathrm{GPa}^{17}$ and its predicted value is $27.3 \mathrm{GPa}\left(\mathrm{Gd}_{63.7} \mathrm{Y}_{9.08} \mathrm{Al}_{18.15} \mathrm{Co}_{9.08}\right)$.

The Poisson's ratio estimations for twenty different alloys were also calculated and compared with those reported in the literature. The corresponding alloys are listed in Table 7; it also includes the elastic constants ratios $\left(c_{44} / c_{11}\right.$ and $\left.c_{12} / c_{11}\right)$ calculated by the database. The absolute difference percentage 

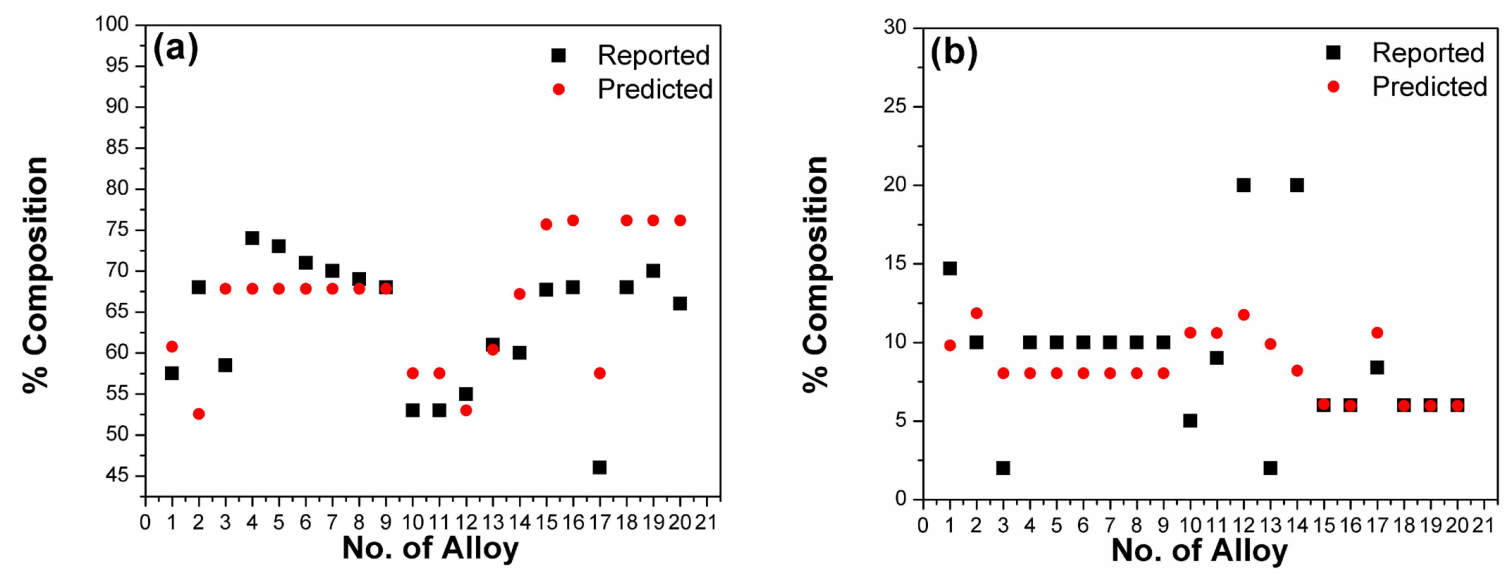

Figure 3. Difference of chemical compositions of ternary alloys. (a) Composition predictions of solvents for twenty different quaternary alloys and (b) Difference of quaternary large solutes for predicted and calculated alloys.

Table 5. Predicted and reported $\mathrm{G}$ for several alloys; alloys 1-12 ${ }^{17}$ and $13-20^{13}$

\begin{tabular}{|c|c|c|c|c|}
\hline$N^{\circ}$. alloy & Reported composition & Predicted composition & Reported $G$ (GPa) & Predicted $G(\mathrm{GPa})$ \\
\hline 1 & $\mathrm{Cu}_{60} \mathrm{Zr}_{20} \mathrm{Hf}_{10} \mathrm{Ti}_{10}$ & $\mathrm{Cu}_{70.99} \mathrm{Zr}_{14.51} \mathrm{Hf}_{7.25} \mathrm{Ti}_{7.25}$ & 36.9 & 44.2 \\
\hline 2 & $\mathrm{Mg}_{65} \mathrm{Cu}_{25} \mathrm{~Tb}_{10}$ & $\mathrm{Mg}_{79.03} \mathrm{Cu}_{10.48} \mathrm{~Tb}_{10.48}$ & 19.6 & 20.8 \\
\hline 3 & $\mathrm{Gd}_{40} \mathrm{Y}_{16} \mathrm{Al}_{24} \mathrm{Co}_{20}$ & $\mathrm{Gd}_{63.7} \mathrm{Y}_{9.08} \mathrm{Al}_{18.15} \mathrm{Co}_{9.08}$ & 23.5 & 27.3 \\
\hline 4 & $\mathrm{Er}_{50} \mathrm{Al}_{24} \mathrm{Co}_{20} \mathrm{Y}_{6}$ & $\mathrm{Er}_{64.61} \mathrm{Al}_{17.7} \mathrm{Co}_{8.85} \mathrm{Y}_{8.85}$ & 27 & 31.8 \\
\hline 5 & $\mathrm{Ca}_{65} \mathrm{Mg}_{15} \mathrm{Zn}_{20}$ & $\mathrm{Ca}_{69.44} \mathrm{Mg}_{15.28} \mathrm{Zn}_{15.28}$ & 10.1 & 14.3 \\
\hline 6 & $\mathrm{Ho}_{39} \mathrm{Al}_{25} \mathrm{Co}_{20} \mathrm{Y}_{16}$ & $\mathrm{Ho}_{64.4} \mathrm{Al}_{17.8} \mathrm{Co}_{8.9} \mathrm{Y}_{8.9}$ & 26.2 & 30.5 \\
\hline 7 & $\mathrm{Ho}_{55} \mathrm{Al}_{25} \mathrm{Co}_{20}$ & $\mathrm{Ho}_{51.61} \mathrm{Al}_{32.26} \mathrm{Co}_{16.13}$ & 25.42 & 34.1 \\
\hline 8 & $\mathrm{Er}_{55} \mathrm{Al}_{25} \mathrm{Co}_{20}$ & $\mathrm{Er}_{56.25} \mathrm{Al}_{29.17} \mathrm{Co}_{14.58}$ & 27.08 & 34.4 \\
\hline 9 & $\mathrm{Mg}_{65} \mathrm{Cu}_{25} \mathrm{Gd}_{10}$ & $\mathrm{Mg}_{80.65} \mathrm{Cu}_{9.68} \mathrm{Gd}_{9.68}$ & 19.3 & 20.5 \\
\hline 10 & $\mathrm{Mg}_{65} \mathrm{Cu}_{25} \mathrm{Y}_{10}$ & $\mathrm{Mg}_{80.65} \mathrm{Cu}_{9.68} \mathrm{Y}_{9.68}$ & 19.4 & 20.8 \\
\hline 11 & $\mathrm{Ca}_{65} \mathrm{Li}_{9.96} \mathrm{Mg}_{8.54} \mathrm{Zn}_{16.5}$ & $\mathrm{Ca}_{53.7} \mathrm{Li}_{11.58} \mathrm{Mg}_{11.58} \mathrm{Zn}_{23.15}$ & 8.95 & 16.4 \\
\hline 12 & $\mathrm{Ni}_{60} \mathrm{Nb}_{35} \mathrm{Sn}_{5}$ & $\mathrm{Ni}_{71.36} \mathrm{Nb}_{21.48} \mathrm{Sn}_{7.16}$ & 66.3 & 63.7 \\
\hline 13 & $\mathrm{Mg}_{58.8} \mathrm{Cu}_{30.5} \mathrm{Y}_{11}$ & $\mathrm{Mg}_{80.65} \mathrm{Cu}_{9.68} \mathrm{Y}_{9.68}$ & 20.4 & 20.8 \\
\hline 14 & $\mathrm{Fe}_{65} \mathrm{Mo}_{14} \mathrm{C}_{15} \mathrm{~B}_{6}$ & $\mathrm{Fe}_{66.99} \mathrm{Mo}_{8.25} \mathrm{C}_{16.51} \mathrm{~B}_{8.25}$ & 73 & 79 \\
\hline 15 & $\mathrm{Zr}_{46.25} \mathrm{Cu}_{46.25} \mathrm{Al}_{7.5}$ & $\mathrm{Zr}_{56.81} \mathrm{Cu}_{32.39} \mathrm{Al}_{10.8}$ & 34.3 & 37.1 \\
\hline 16 & $\mathrm{Mg}_{57} \mathrm{Cu}_{31} \mathrm{Y}_{6.6} \mathrm{Nd}_{5.4}$ & $\mathrm{Mg}_{67.84} \mathrm{Cu}_{16.08} \mathrm{Y}_{8.04} \mathrm{Nd}_{8.04}$ & 20.7 & 22.6 \\
\hline 17 & $\mathrm{Hf}_{50} \mathrm{Ni}_{25} \mathrm{Al}_{25}$ & $\mathrm{Hf}_{60} \mathrm{Ni}_{30} \mathrm{Al}_{10}$ & 47 & 43.4 \\
\hline 18 & $\mathrm{Hf}_{55} \mathrm{Ni}_{25} \mathrm{Al}_{20}$ & $\mathrm{Hf}_{60} \mathrm{Ni}_{30} \mathrm{Al}_{10}$ & 43.7 & 43.4 \\
\hline 19 & $\mathrm{Zr}_{45.25} \mathrm{Cu}_{46.25} \mathrm{Al}_{7.5} \mathrm{Sn}_{1}$ & $\mathrm{Zr}_{45.25} \mathrm{Cu}_{46.25} \mathrm{Al}_{7.5} \mathrm{Sn}_{1}$ & 35.7 & 42.2 \\
\hline 20 & $\mathrm{Zr}_{64} \mathrm{Cu}_{26} \mathrm{Al}_{10}$ & $\mathrm{Zr}_{63.68} \mathrm{Cu}_{24.21} \mathrm{Al}_{12.11}$ & 28.7 & 35.8 \\
\hline
\end{tabular}

of the Poisson's ratio in most alloys compared in this work was between $3.8 \%-12 \%$. Compositions 3,7 , and 8 , show smaller values than $3 \%$, due to chemical composition similarity. Compositions 10 and 17 , show a greater value than $13 \%$, due to greater discrepancy between reported and calculated compositions.

Figure 5 shows a Blackman diagram constructed using the data from twenty different predicted alloys (listed in Table 7), with the Poisson's ratio. Concerning to the intrinsic toughness estimated for the twenty different predicted alloys, the Poisson's ratio was consistent with the ductile behaviour in most of the alloys. These results could provide an insight into the tough-brittle behaviour that the system might present. Those values at the upper left, such as: $\mathrm{Pd}_{75} \mathrm{Cu}_{12.5} \mathrm{Si}_{12.5}(0.216$, $0.676,0.403 ; c_{44} / c_{11}, c_{12} / c_{11}$, Poisson's ratio, respectively) and $\mathrm{Ni}_{71.36} \mathrm{Nb}_{21.48} \mathrm{Sn}_{7.16}(0.274,0.590,0.371)$ corresponds to higher Poisson's ratio values (low $c_{44} / c_{11}$ and high $c_{12} / c_{11}$ ), and so those systems would be expected to display ductile behaviour.

The Poisson's ratio reported and predicted for $\mathrm{Ho}_{39} \mathrm{Al}_{25} \mathrm{Co}_{20} \mathrm{Y}_{16}$ are 0.319 and 0.328 , respectively. These are down to the right on the Blackman construction and the transition from brittle to tough behaviour would be expected at $v \sim 0.32$, which is consistent with the criterion reported. ${ }^{19}$ 
Table 6. Predicted and reported E for several alloys; alloys 1-12 ${ }^{17}$ and $13-20^{13}$.

\begin{tabular}{|c|c|c|c|c|}
\hline $\mathbf{N}^{\circ}$. alloy & Reported composition & Predicted composition & Reported E (GPa) & Predicted E (GPa) \\
\hline 1 & $\mathrm{Cu}_{60} \mathrm{Zr}_{20} \mathrm{Hf}_{10} \mathrm{Ti}_{10}$ & $\mathrm{Cu}_{70.99} \mathrm{Zr}_{14.51} \mathrm{Hf}_{7.25} \mathrm{Ti}_{7.25}$ & 101.1 & 117.7 \\
\hline 2 & $\mathrm{Mg}_{65} \mathrm{Cu}_{25} \mathrm{~Tb}_{10}$ & $\mathrm{Mg}_{79.03} \mathrm{Cu}_{10.48} \mathrm{~Tb}_{10.48}$ & 51.3 & 54.8 \\
\hline 3 & $\mathrm{Gd}_{40} \mathrm{Y}_{16} \mathrm{Al}_{24} \mathrm{Co}_{20}$ & $\mathrm{Gd}_{63.7} \mathrm{Y}_{9.08} \mathrm{Al}_{18.15} \mathrm{Co}_{9.08}$ & 62.2 & 70.3 \\
\hline 4 & $\mathrm{Er}_{50} \mathrm{Al}_{24} \mathrm{Co}_{20} \mathrm{Y}_{6}$ & $\mathrm{Er}_{64.61} \mathrm{Al}_{17.7} \mathrm{Co}_{8.85} \mathrm{Y}_{8.85}$ & 71.1 & 81.7 \\
\hline 5 & $\mathrm{Ho}_{39} \mathrm{Al}_{25} \mathrm{Co}_{20} \mathrm{Y}_{16}$ & $\mathrm{Ho}_{64.4} \mathrm{Al}_{17.8} \mathrm{Co}_{8.9} \mathrm{Y}_{8.9}$ & 69.1 & 78.4 \\
\hline 6 & $\mathrm{Tm}_{39} \mathrm{Zr}_{15} \mathrm{Al}_{25} \mathrm{Co}_{20}$ & $\mathrm{Tm}_{64.65} \mathrm{Zr}_{8.84} \mathrm{Al}_{17.68} \mathrm{Co}_{8.84}$ & 75 & 86.5 \\
\hline 7 & $\mathrm{Yb}_{62.5} \mathrm{Zn}_{15} \mathrm{Mg}_{17.5} \mathrm{Cu}_{5}$ & $\mathrm{Yb}_{54.13} \mathrm{Zn}_{11.47} \mathrm{Mg}_{22.94} \mathrm{Cu}_{11.47}$ & 26.5 & 50.3 \\
\hline 8 & $\operatorname{Pr}_{55} \mathrm{Al}_{25} \mathrm{Co}_{20}$ & $\operatorname{Pr}_{56.25} \mathrm{Al}_{29.17} \mathrm{Co}_{14.58}$ & 45.9 & 71.9 \\
\hline 9 & $\mathrm{Ho}_{55} \mathrm{Al}_{25} \mathrm{Co}_{20}$ & $\mathrm{Ho}_{51.61} \mathrm{Al}_{32.26} \mathrm{Co}_{16.13}$ & 66.64 & 89 \\
\hline 10 & $\mathrm{Er}_{55} \mathrm{Al}_{25} \mathrm{Co}_{20}$ & $\mathrm{Er}_{56.25} \mathrm{Al}_{29.17} \mathrm{Co}_{14.58}$ & 70.72 & 90.2 \\
\hline 11 & $\mathrm{Mg}_{65} \mathrm{Cu}_{25} \mathrm{Gd}_{10}$ & $\mathrm{Mg}_{80.65} \mathrm{Cu}_{9.68} \mathrm{Gd}_{9.68}$ & 50.6 & 54 \\
\hline 12 & $\mathrm{Ca}_{65} \mathrm{Li}_{9.96} \mathrm{Mg}_{8.54} \mathrm{Zn}_{16.5}$ & $\mathrm{Ca}_{53.7} \mathrm{Li}_{11.58} \mathrm{Mg}_{11.58} \mathrm{Zn}_{23.15}$ & 23.4 & 41.5 \\
\hline 13 & $\mathrm{Mg}_{58.8} \mathrm{Cu}_{30.5} \mathrm{Y}_{11}$ & $\mathrm{Mg}_{80.65} \mathrm{Cu}_{9.68} \mathrm{Y}_{9.68}$ & 53.9 & 54 \\
\hline 14 & $\mathrm{Zr}_{46.25} \mathrm{Cu}_{46.25} \mathrm{Al}_{7.5}$ & $\mathrm{Zr}_{56.81} \mathrm{Cu}_{32.39} \mathrm{Al}_{10.8}$ & 93.81 & 99 \\
\hline 15 & $\mathrm{Mg}_{57} \mathrm{Cu}_{31} \mathrm{Y}_{6.6} \mathrm{Nd}_{5.4}$ & $\mathrm{Mg}_{67.84} \mathrm{Cu}_{16.08} \mathrm{Y}_{8.04} \mathrm{Nd}_{8.04}$ & 54.4 & 59.5 \\
\hline 16 & $\mathrm{Hf}_{50} \mathrm{Ni}_{25} \mathrm{Al}_{25}$ & $\mathrm{Hf}_{60} \mathrm{Ni}_{30} \mathrm{Al}_{10}$ & 125.6 & 113.8 \\
\hline 17 & $\mathrm{Cu}_{47} \mathrm{Zr}_{47} \mathrm{Al}_{6}$ & $\mathrm{Cu}_{67.57} \mathrm{Zr}_{24.32} \mathrm{Al}_{8.11}$ & 92.4 & 113.6 \\
\hline 18 & $\mathrm{Hf}_{55} \mathrm{Ni}_{25} \mathrm{Al}_{20}$ & $\mathrm{Hf}_{60} \mathrm{Ni}_{30} \mathrm{Al}_{10}$ & 117.63 & 113.8 \\
\hline 19 & $\mathrm{Zr}_{45.25} \mathrm{Cu}_{46.25} \mathrm{Al}_{7.5} \mathrm{Sn}_{1}$ & $\mathrm{Zr}_{45.25} \mathrm{Cu}_{46.25} \mathrm{Al}_{7.5} \mathrm{Sn}_{1}$ & 97.3 & 112.8 \\
\hline 20 & $\mathrm{Zr}_{64} \mathrm{Cu}_{26} \mathrm{Al}_{10}$ & $\mathrm{Zr}_{63.68} \mathrm{Cu}_{24.21} \mathrm{Al}_{12.11}$ & 78.85 & 95.5 \\
\hline
\end{tabular}
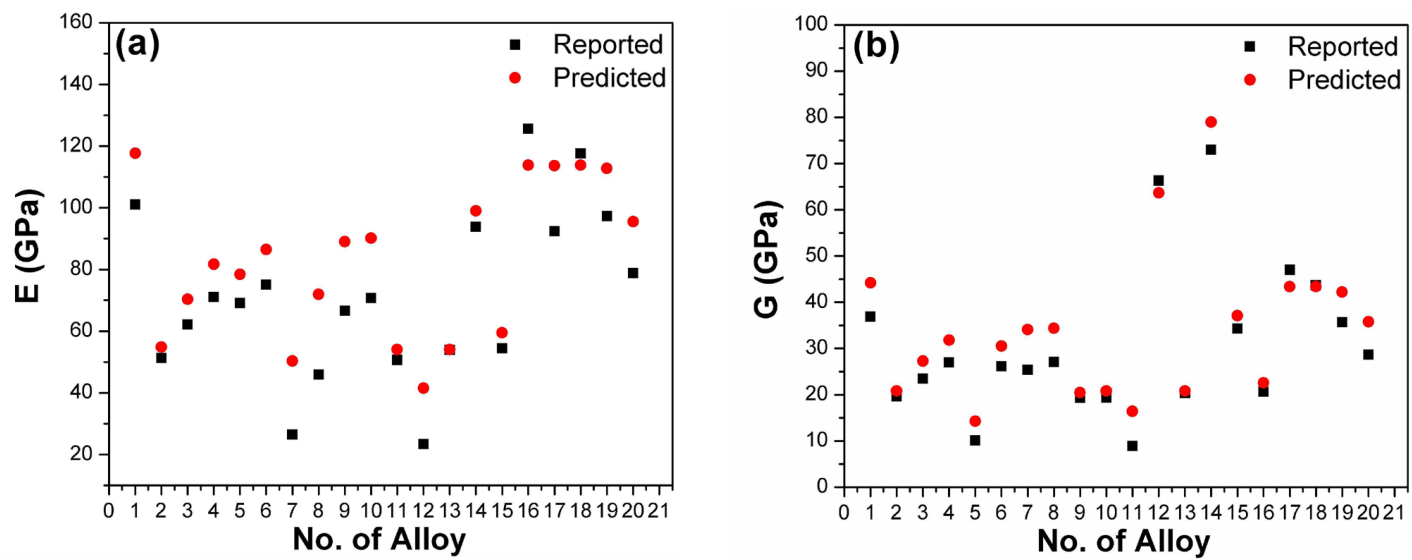

Figure 4. Predicted and reported elastic proprieties values for several alloys. (a) Elastic modulus and (b) Shear modulus.

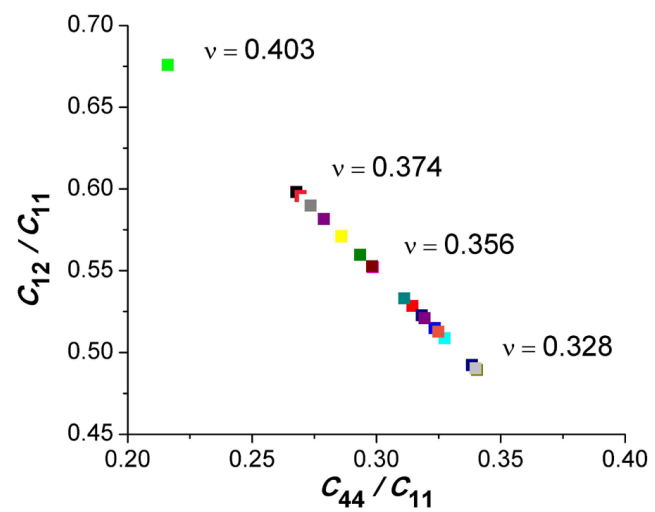

Figure 5. Blackman diagram of twenty different predicted alloys.
With the database, it was possible to make other predictions based on elastic properties, such as the kinetic fragility index, $m$, which was calculated with equation $(4)^{39}$.

$m=12(K / G+0.67)$

The kinetic fragility index is recently used to know whether an alloy will present high or low GFA ${ }^{63}$. Since elastic moduli can be potentially correlated to a wide range of physical, mechanical and thermal properties in BMGs, the way the database has been used here may be considered a starting point for how it can be implemented in future studies. Additionally, the database performs three different composition predictions for the ternary systems, the one that considers the gamma sites to be empty, another in which gamma sites are half occupied by $\beta_{\gamma}$ anti-site defects and 
Table 7. Poisson's ratios for different alloys ${ }^{17}$, and their respectively predicted elastic constants ratios.

\begin{tabular}{|c|c|c|c|c|c|c|c|}
\hline $\begin{array}{l}N^{\circ} \text {. } \\
\text { alloy }\end{array}$ & $\begin{array}{c}\text { Reported } \\
\text { composition }\end{array}$ & $\begin{array}{l}\text { Predicted } \\
\text { composition }\end{array}$ & Reported $v$ & Predicted $v$ & $\begin{array}{c}v \\
\% \text { Difference } \\
\text { ABS }\end{array}$ & $\begin{array}{c}\text { Predicted } \\
c_{12} / c_{11}\end{array}$ & $\begin{array}{c}\text { Predicted } \\
c_{44} / c_{11}\end{array}$ \\
\hline 1 & $\mathrm{Zr}_{65} \mathrm{Al}_{10} \mathrm{Ni}_{10} \mathrm{Cu}_{15}$ & $\mathrm{Zr}_{57.19} \mathrm{Al}_{10.7} \mathrm{Ni}_{10.7} \mathrm{Cu}_{21.41}$ & 0.355 & 0.374 & 5.1 & 0.598 & 0.268 \\
\hline 2 & $\mathrm{Nd}_{60} \mathrm{Al}_{10} \mathrm{Co}_{10} \mathrm{Fe}_{20}$ & $\mathrm{Nd}_{56.36} \mathrm{Al}_{10.91} \mathrm{Co}_{10.91} \mathrm{Fe}_{21.82}$ & 0.306 & 0.346 & 11.6 & 0.528 & 0.315 \\
\hline 3 & $\mathrm{Pd}_{77.5} \mathrm{Cu}_{6} \mathrm{Si}_{16.5}$ & $\mathrm{Pd}_{75} \mathrm{Cu}_{12.5} \mathrm{Si}_{12.5}$ & 0.411 & 0.403 & 2.0 & 0.676 & 0.216 \\
\hline 4 & $\mathrm{Gd}_{40} \mathrm{Y}_{16} \mathrm{Al}_{24} \mathrm{Co}_{20}$ & $\mathrm{Gd}_{63.7} \mathrm{Y}_{9.08} \mathrm{Al}_{18.15} \mathrm{Co}_{9.08}$ & 0.321 & 0.340 & 5.6 & 0.515 & 0.324 \\
\hline 5 & $\mathrm{Ca}_{65} \mathrm{Mg}_{15} \mathrm{Zn}_{20}$ & $\mathrm{Ca}_{69.44} \mathrm{Mg}_{15.28} \mathrm{Zn}_{15.28}$ & 0.300 & 0.337 & 11.0 & 0.509 & 0.328 \\
\hline 6 & $\mathrm{Ce}_{70} \mathrm{Al}_{10} \mathrm{Ni}_{10} \mathrm{Cu}_{10}$ & $\mathrm{Ce}_{52.56} \mathrm{Al}_{11.86} \mathrm{Ni}_{23.72} \mathrm{Cu}_{11.86}$ & 0.313 & 0.356 & 12.1 & 0.552 & 0.299 \\
\hline 7 & $\mathrm{Pr}_{60} \mathrm{Al}_{10} \mathrm{Ni}_{10} \mathrm{Cu}_{20}$ & $\operatorname{Pr}_{56.14} \mathrm{Al}_{10.97} \mathrm{Ni}_{10.97} \mathrm{Cu}_{21.93}$ & 0.360 & 0.363 & 0.8 & 0.571 & 0.286 \\
\hline 8 & $\mathrm{Ho}_{39} \mathrm{Al}_{25} \mathrm{Co}_{20} \mathrm{Y}_{16}$ & $\mathrm{Ho}_{64.4} \mathrm{Al}_{17.8} \mathrm{Co}_{8.9} \mathrm{Y}_{8.9}$ & 0.319 & 0.328 & 2.7 & 0.489 & 0.341 \\
\hline 9 & $\mathrm{Tm}_{40} \mathrm{Zr}_{15} \mathrm{Al}_{25} \mathrm{Co}_{20}$ & $\mathrm{Tm}_{64.65} \mathrm{Zr}_{8.84} \mathrm{Al}_{17.68} \mathrm{Co}_{8.84}$ & 0.307 & 0.330 & 7.0 & 0.492 & 0.339 \\
\hline 10 & $\mathrm{Yb}_{62.5} \mathrm{Zn}_{15} \mathrm{Mg}_{17.5} \mathrm{Cu}_{5}$ & $\mathrm{Yb}_{54.13} \mathrm{Zn}_{11.47} \mathrm{Mg}_{22.94} \mathrm{Cu}_{11.47}$ & 0.276 & 0.368 & 25.0 & 0.582 & 0.279 \\
\hline 11 & $\mathrm{La}_{55} \mathrm{Al}_{25} \mathrm{Co}_{20}$ & $\mathrm{La}_{61.54} \mathrm{Al}_{19.23} \mathrm{Co}_{19.23}$ & 0.327 & 0.356 & 8.1 & 0.553 & 0.298 \\
\hline 12 & $\mathrm{Pr}_{55} \mathrm{Al}_{25} \mathrm{Co}_{20}$ & $\operatorname{Pr}_{56.25} \mathrm{Al}_{29.17} \mathrm{Co}_{14.58}$ & 0.324 & 0.359 & 9.7 & 0.560 & 0.294 \\
\hline 13 & $\mathrm{~Tb}_{55} \mathrm{Al}_{25} \mathrm{Co}_{20}$ & $\mathrm{~Tb}_{61.54} \mathrm{Al}_{19.23} \mathrm{Co}_{19.23}$ & 0.302 & 0.347 & 13.0 & 0.533 & 0.311 \\
\hline 14 & $\mathrm{Dy}_{55} \mathrm{Al}_{25} \mathrm{Co}_{20}$ & $\mathrm{Dy}_{61.54} \mathrm{Al}_{19.23} \mathrm{Co}_{19.23}$ & 0.304 & 0.343 & 11.4 & 0.523 & 0.318 \\
\hline 15 & $\mathrm{Ho}_{55} \mathrm{Al}_{25} \mathrm{Co}_{20}$ & $\mathrm{Ho}_{61.54} \mathrm{Al}_{19.23} \mathrm{Co}_{19.23}$ & 0.311 & 0.340 & 8.5 & 0.513 & 0.325 \\
\hline 16 & $\mathrm{Er}_{55} \mathrm{Al}_{25} \mathrm{Co}_{20}$ & $\mathrm{Er}_{56.25} \mathrm{Al}_{29.17} \mathrm{Co}_{14.58}$ & 0.306 & 0.343 & 10.8 & 0.521 & 0.319 \\
\hline 17 & $\mathrm{Mg}_{65} \mathrm{Cu}_{25} \mathrm{Gd}_{10}$ & $\mathrm{Mg}_{67.57} \mathrm{Cu}_{24.32} \mathrm{Gd}_{8.11}$ & 0.310 & 0.373 & 16.9 & 0.596 & 0.270 \\
\hline 18 & $\mathrm{Mg}_{65} \mathrm{Cu}_{25} \mathrm{Y}_{10}$ & $\mathrm{Mg}_{67.57} \mathrm{Cu}_{24.32} \mathrm{Y}_{8.11}$ & 0.329 & 0.372 & 11.6 & 0.593 & 0.271 \\
\hline 19 & $\mathrm{Ca}_{65} \mathrm{Li}_{9.96} \mathrm{Mg}_{8.54} \mathrm{Zn}_{16.5}$ & $\mathrm{Ca}_{53.7} \mathrm{Li}_{11.58} \mathrm{Mg}_{11.58} \mathrm{Zn}_{23.15}$ & 0.307 & 0.329 & 6.7 & 0.490 & 0.340 \\
\hline 20 & $\mathrm{Ni}_{60} \mathrm{Nb}_{35} \mathrm{Sn}_{5}$ & $\mathrm{Ni}_{71.36} \mathrm{Nb}_{21.48} \mathrm{Sn}_{7.16}$ & 0.385 & 0.371 & 3.8 & 0.590 & 0.274 \\
\hline
\end{tabular}

the last one that assumes all gamma sites are occupied by the presence of $\beta$ anti-site defects. Thus, the best prediction approach was chosen to be compared with the reported data in the ternary systems.

\subsection{Experimental results}

In order to test the database, some glass alloys in the form of bulk were experimentally obtained. Figure 6 shows the XRD pattern of the $\mathrm{Zr}_{57.52} \mathrm{Ag}_{10.62} \mathrm{Al}_{10.62} \mathrm{Co}_{21.24}, \mathrm{Zr}_{57.19} \mathrm{Al}_{10.7} \mathrm{Ni}_{10.7} \mathrm{Cu}_{21.41}$, and $\mathrm{Hf}_{60.22} \mathrm{Al}_{9.95} \mathrm{Cu}_{9.95} \mathrm{Ni}_{19.89}$ alloys. The XRD patterns are constituted by a single broad peak (located between $2 \theta$ 35 and $55^{\circ}$ ) typical of a metallic glass. These results confirm the usefulness of the work presented in this manuscript, where it was possible to design, model and produce bulk metallic glasses a-priori, reducing the experimental work time associated with standard experimental processes.

Table 8 shows packing efficiency values and kinetic fragility index $m$ of alloys produced. The efficiency packing values were between $45 \%$ and 50\%. According to Angell ${ }^{61-62}$, glass-forming liquids can be classified into strong and fragile liquids, depending on their fragility. The upper and lower limits of parameter are theoretically estimated between 16 for 'strong' systems and 200 for 'fragile' systems ${ }^{63}$. The alloys obtained are strong systems with high glass forming ability.

Table 9 shows compositions calculated by "mixing rules", elastic properties, and elastic constants ratios $\left(c_{44} / c_{11}\right.$ and $\left.c_{12} / c_{11}\right)$. The typical BMGs have Young's modulus $\mathrm{E} \sim 25 \mathrm{GPa}-250 \mathrm{GPa}$, shear modulus $\mathrm{G} \sim 9 \mathrm{GPa}-88 \mathrm{GPa}$, and bulk modulus $\mathrm{K} \sim 20 \mathrm{GPa}-243 \mathrm{GPa}{ }^{17}$.

A good correlation between microhardness, $\mathrm{H}_{v}$, and Young's modulus, E, has been reported elsewhere ${ }^{17}$, in that work, it is reported an $\mathrm{E} / \mathrm{H}_{\mathrm{v}} \sim 20$.

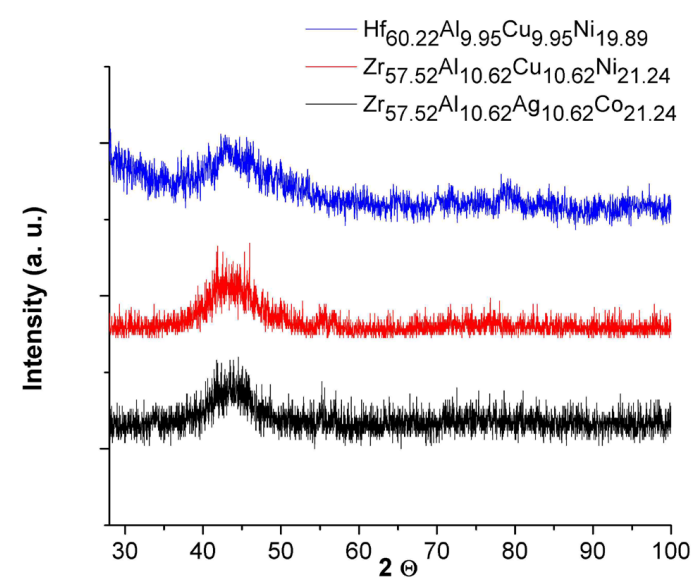

Figure 6. XRD patterns of the experimentally obtained bulk metallic glasses.

The microhardness values obtained experimentally in this work of $\mathrm{Zr}_{57.52} \mathrm{Ag}_{10.62} \mathrm{Al}_{10.62} \mathrm{Co}_{21.24}, \mathrm{Zr}_{57.19} \mathrm{Al}_{10.7} \mathrm{Ni}_{10.7} \mathrm{Cu}_{21.41}$ and $\mathrm{Hf}_{60.22} \mathrm{Al}_{9.95} \mathrm{Cu}_{9.95} \mathrm{Ni}_{19.89}$ alloys, are 3.8, 4.0 and $3.6 \mathrm{GPa}$, respectively. If these values are substituted in the $\mathrm{E} / \mathrm{H}_{\mathrm{v}}=20$ relationship found in the previous research ${ }^{17}$, then the Young's modulus values calculated are 76, 80 and $73 \mathrm{GPa}$. These values are closed to the calculated values with the "mixing rules" (table 9).

The elastic constants ratios were used to plot a Blackman diagram. Figure 7 shows the Blackman diagram of alloys mentioned above with their corresponding Poisson's ratio. 
Table 8. Compositions, packaging efficiency and kinetic index of fragility, calculated with the database for the obtained BMGs.

\begin{tabular}{ccc}
\hline Alloy & Efficiency packing \% & Kinetic fragility index, $\boldsymbol{m}$ \\
\hline $\mathrm{Zr}_{57.52} \mathrm{Ag}_{10.62} \mathrm{Al}_{10.62} \mathrm{Co}_{21.24}$ & 45.37 & 40.1 \\
$\mathrm{Zr}_{57.19} \mathrm{Al}_{10.7} \mathrm{Ni}_{10.7} \mathrm{Cu}_{21.41}$ & 46.56 & 40.8 \\
$\mathrm{Hf}_{60.22} \mathrm{Al}_{9.95} \mathrm{Cu}_{9.95} \mathrm{Ni}_{19.89}$ & 49.8 & 46 \\
\hline
\end{tabular}

Table 9. Elastic properties calculated with the "mixing rules" for the obtained BMGs.

\begin{tabular}{cccccc}
\hline \multirow{2}{*}{ Alloy } & \multicolumn{3}{c}{ Elastic proprieties (GPa) } & \multicolumn{3}{c}{ Elastic constants ratios } \\
\cline { 2 - 7 } & $\mathbf{E}$ & $\mathbf{G}$ & $\mathbf{K}$ & $\mathbf{c}_{\mathbf{1 2}} / \mathbf{c}_{\mathbf{1 1}}$ & $\mathbf{c}_{44} / \mathbf{c}_{\mathbf{1 1}}$ \\
\hline $\mathrm{Zr}_{57.52} \mathrm{Ag}_{10.62} \mathrm{Al}_{10.62} \mathrm{Co}_{21.24}$ & 71.4 & 40.9 & 109.3 & 0.591 & 0.272 \\
$\mathrm{Zr}_{57.19} \mathrm{Al}_{10.7} \mathrm{Ni}_{10.7} \mathrm{Cu}_{21.41}$ & 83.8 & 40.1 & 109.5 & 0.597 & 0.268 \\
$\mathrm{Hf}_{60.22} \mathrm{Al}_{9.95} \mathrm{Cu}_{9.95} \mathrm{Ni}_{19.89}$ & 77.8 & 37.8 & 119.5 & 0.640 & 0.240 \\
\hline
\end{tabular}

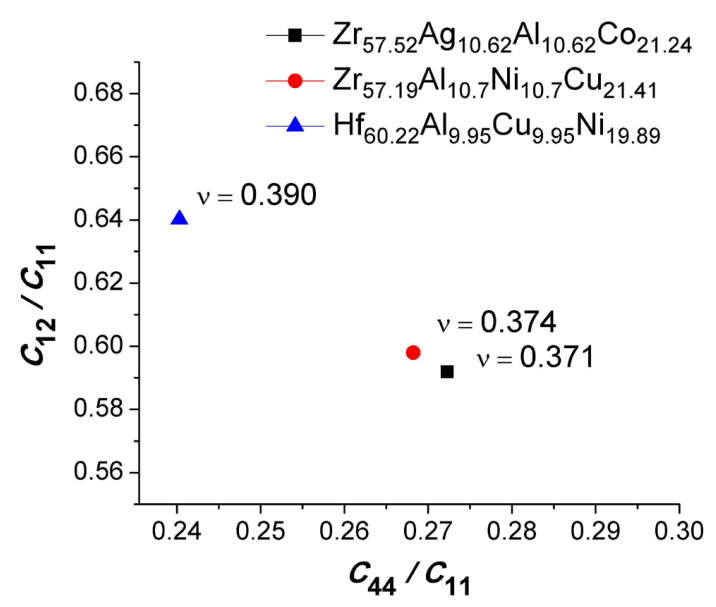

Figure 7. Blackman diagram of the experimentally obtained bulk metallic glasses.

It shows the values at the upper left (low $c_{44} / c_{11}$ and high $c_{12} / c_{11}$ ) and high Poisson's ratio values, suggest ductile behaviour.

\section{Conclusions}

The results of the database presented here for estimating composition and elemental elastic properties in metallic glasses have been able to closely approach those experimentally

\section{References}

1. Goldschmidt VM. Geochemische Verteilungsgesetze der Elemente VIII. Oslo: Skrifter Norske Videnkaps; 1926.

2. Zachariasen WH. The atomic arrangement in glass. Journal of the American Chemical Society. 1932;54(10):3841-3851. DOI: $10.1021 / \mathrm{ja} 01349 \mathrm{a} 006$

3. Bernal JD, Mason J. Packing of spheres: coordination of randomly packed spheres. Nature. 1960;188(4754):910-911. DOI: $10.1038 / 188910 \mathrm{a} 0$

4. Miracle DB. A structural model for metallic glasses. Nature Materials. 2004;3:697-702. doi:10.1038/nmat1219

5. Sun KH. Fundamental condition of glass formation. Journal of the American Ceramic Society. 1947;30(9):277-281. DOI: 10.1111/j.1151-2916.1947.tb19654.x determined and reported in the literature. They additionally provide valuable information regarding the mechanical behaviour that these alloys might present. This illustrates the usefulness of these theoretical models. In relation to the packing efficiency calculations, particularly those obtained for the quaternary systems, it can be noticed that some other factors such as chemical affinity, bonding and/or the enthalpies of mixing, must play a key role in the BMG-forming systems. The database enclosing the methods used in this work has been developed; incorporates the elemental elastic constants for the most common alloying elements as well as their atomic radii information. The quaternary alloys experimentally obtained are strong systems with high glass forming ability, which is consistent with the Miracle's model and kinetic fragility index $m$. The Young's modulus estimated with microhardness values are closed to those calculated by the "mixing rules". The Poisson's ratio and Blackman diagram, suggest ductile behaviour of the BMGs obtained.

\section{Acknowledgements}

The authors would like to acknowledge the financial support from SEP CONACYT 178289 for funding the project. A. Tejeda-Cruz, S. García-Galán, J. MoralesRosales, C. Flores-Morales, C. Delgado, G. Aramburo, O. Novelo and J. M. Garcia-León are also acknowledged for their technical support.

6. Polk D. The structure of glassy metallic alloys. Acta Metallurgica. 1972;20(4):485-491. Doi: 10.1016/0001-6160(72)90003-X

7. Egami T, Waseda YJ. Atomic size effect on the formability of metallic glasses. Journal of Non-Crystalline Solids. 1984;64(12):113-134.

8. Granato AV. Thermodynamic properties of liquid and amorphous monatomic metals from an interstitialcy model. Journal of Non-Crystalline Solids. 1993;156-158(Part 1):402-406. DOI: 10.1016/0022-3093(93)90206-D

9. Egami T. Universal criterion for metallic glass formation. Materials Science and Engineering. 1997;226-228:261-267. doi:10.1016/S0921-5093(97)80041-X

10. Senkov ON, Miracle DB. Effect of the atomic size distribution on glass forming ability of amorphous metallic alloys. Materials 
Research Bulletin. 2001;36(12):2183-2198. DOI: 10.1016/ S0025-5408(01)00715-2

11. Egami T. Atomistic mechanism of bulk metallic glass formation. Journal of Non-Crystalline Solids. 2003;317(1-2):30-33. doi:10.1016/S0022-3093(02)02003-3

12. Axinte E. Metallic glasses from "alchemy" to pure science: Present and future of design, processing and applications of glassy metals. Materials and Design. 2012;35:518-556. DOI: $10.1016 /$ j.matdes.2011.09.028

13. Plummer JD, Todd I. Implications of elastic constants, fragility, and bonding on permanent deformation in metallic glass. Appllied Physics Letters. 2011;98(2):021907. http://dx.doi. org/10.1063/1.3540652

14. Miracle DB, Sanders WS, Senkov O. The influence of efficient atomic packing on the constitution of metallic glasses. Philosophical Magazine. 2003;83(20):2409-2428. DOI: $10.1080 / 1478643031000098828$

15. Miracle DB. The efficient cluster packing model - An atomic structural model for metallic glasses. Acta Materialia. 2006;54(16):4317-4336. DOI: 10.1016/j.actamat.2006.06.002

16. Liu GR. A step-by-step method of rule-of-mixture of fiber and particle-reinforced composite materials. Composite Structure.1998;40(3-4):313-322. doi:10.1016/S02638223(98)00033-6

17. Wang WH. The elastic properties, elastic models and elastic perspectives of metallic glasses. Progress in Materials Science. 2012;57:487-656.

18. Ledbetter H. Handbook of elastic properties of solids liquids and gases. New York: Academic Science; 2001. Vol. 2

19. Lewandowski J, Wang WH, Greer AL. Intrinsic plasticity or brittleness of metallic glasses. Philosophical Magazine Letters. 2005;85(2):77-87.

20. Yao KF, Chen N. Pd-Si binary bulk metallic glass. Science in China Series G: Physics, Mechanics and Astronomy. 2008;51(4):414-420. DOI: 10.1007/s11433-008-0051-4

21. Guo FQ, Poon SJ, Shiflet GJ. CaAl-based bulk metallic glasses with high thermal stability. Appllied Physics Letters. 2004;84(1):37-39. http://dx.doi.org/10.1063/1.1637940

22. Leonhardt M, Löser W, Lindenkreuz HG. Solidification kinetics and phase formation of undercooled eutectic Ni-Nb melts. Acta Materialia. 1999;47(10):2961-2968.

23. Wang D, Li Y, Sun BB, Sui ML, Lu K, Ma E. Bulk metallic glass formation in the binary $\mathrm{Cu}-\mathrm{Zr}$ system. Applied Physics Letters. 2004;84(20): 4029-4031.

24. Buschow $\mathrm{KH}$. Thermal stability of amorphous $\mathrm{Zr}-\mathrm{Cu}$ alloys. Journal of Applied Physics. 1981;52(5):3319-3323.DOI: $10.1063 / 1.329152$

25. WasedaY, Chen HS. A structural study of metallic glasses containing boron (Fe-B, Co-B and Ni-B. Physica Status Solidi.1978;49(1):387-392. DOI: 10.1002/pssa.2210490149

26. Davies HA, Hull JB. Some aspects of splat-quenching in an inert atmosphere and of the formation of non-crystalline phases in Al-17.3 at. \% Cu, germanium and tellurium. Journal of Materials Science. 1974;9:707-717.

27. Nose M, Masumoto T. Characteristics of (Fe, Co and/or Ni)-Zr amorphous alloys. Science Reports of the Research Institute Tohoku University SerA,Physicis, chemistry and metallurgy. 1980;28A:222-236.

28. Chen HS, Jackson KA. The Influence of alloy composition on glass formation and properties. In: Polk DE, Giesen BC. Metallic Glasses. Ohio, USA: Metals Park; 1978. p.74-96.
29. Predecki P, Giessen BC, Grant NJ. New metastable alloy. Transactions of the Metallurgical Society of AIME. 1965;233(7): 1438-1439.

30. Klement W, Willens RH, Duwez P. Non-crystalline structure in solidified gold-silicon alloys. Nature. 1960;187:869-870.

31. Sommer F, Duddek G, Predel B. New glassy alloys. Zeitchrift Metallkude. 1978;69:587-590.

32. St Amand R, Geissen BC. Easy glass formation in simple metal alloys: amorphous metals containing calcium and strontium. Scripta Metallurgica. 1978;12(11):1021-1026. Doi:10.106/00369748(78)90017-0

33. Hasegawa R, Ray R. Iron-boron metallic glasses. Journal of Applied Physics. 1978;49:4174-4178. http://dx.doi. org/10.1063/1.325328

34. Lu S, Tang MB, Xia L. Excellent magnetocaloric effect of a Gd55A120Co25 bulk metallic glass. Physica B: Condensed Matter. 2011;406(18):3398-3401. http://dx.doi.org/10.1016/j. physb.2011.06.006

35. Yu P, Chan KC, Chen W, Xia L. Elastic moduli and mechanical properties of bulk metallic glasses after quasi-static compression. Journal of Alloys and Compounds. 2011;509(34):8518-8521. DOI: 10.1016/j.jallcom.2011.05.113

36. Soubeyroux JL, Puech S, Blandin J. Effect of silver on the glass forming ability of $\mathrm{MgCuGdY}$ bulk metallic glasses. Journal of Alloys and Compounds. 2009;483(1-2) :107-111. DOI : 10.1016/j.jallcom.2008.07.234

37. Peng H Li SS, Qi YP, Huang TY. Mg-Ni-Gd-Ag bulk metallic glass with improved glass-forming ability and mechanical properties. Intermetallics. 2011;19(7):829-832. doi:10.1016/j. intermet.2010.11.029

38. Wada T, Qin FX, Wang XM, Yoshimura M, Inoue A, Sugiyama $\mathrm{N}$, et al. Formation and bioactivation of Zr-Al-Co bulk metallic glasses. Journal of Materials Research. 2009;24(9):2941-2948. DOI: http://dx.doi.org/10.1557/jmr.2009.0348

39. Park ES, Chang HJ, Kim DH. Effect of addition of Be on glassforming ability, plasticity and structural change in $\mathrm{Cu}-\mathrm{Zr}$ bulk metallic glasses. Acta Materialia. 2008;56(13):3120-3131. http://dx.doi.org/10.1016/j.actamat.2008.02.044

40. He Q, Cheng YQ, Ma E, Xu J. Locating bulk metallic glasses with high fracture toughness: Chemical effects and composition optimization. Acta Materialia. 2011;59(1):202-215.doi:10.1016/j. actamat.2010.09.025

41. Zhang L, Cheng YQ, Cao AJ, Xu J, Ma E. Bulk metallic glasses with large plasticity: Composition design from the structural perspective. Acta Materialia. 2009;57 (4) :154-1164.

42. Huang XM, Chang CT, Chang ZY, Wang XD, Cao QP, Shen $\mathrm{BL}$, et al. Formation of bulk metallic glasses in the Fe-MY-B $(\mathrm{M}=$ transition metal) system. Journal of Alloys and Compounds. 2008;460(1-2):708-713. http://dx.doi.org/10.1016/j. jallcom.2007.09.063

43. Guo G, Bian X, Meng Q, Zhao Y, Li X, Zhang S. Ternary Sm-Al-Co bulk metallic glass with high glass-forming ability. Journal of Alloys and Compounds. 2007;431(1-2):167-170. doi:10.1016/j.jallcom.2006.05.072

44. Kong HZ, Li Y, Ding J. Magnetic hardening in amorphous alloy Sm60Fe30A110. Scripta Materialia. 2001;44(5): 829-834. DOI: 10.1016/S1359-6462(00)00652-7

45. Li S, Wang RJ, Pan MX, Zhao DQ, Wang WH. Heavy rare earth based bulk metallic glasses with high thermal stability. Intermetallic. 2006;14:592-595.

46. Jo CL, Xia L, Ding D, Dong YD. Glass formation ability and kinetics of the Gd55A120Ni25 bulk metallic glass. Chinese PhysicsLetters. 2006;23(3):672-674. 
47. Kissinger HE. Reaction kinetics in differential thermal analysis. Analytical Chemistry. 1957;29(11):1702-1706.

48. Zhang B, Zhao DQ, Pan MX, Wang WH, Greer AL. Amorphous metallic plastic. Physical Review Letters. 2005;94:205502

49. Takeuchi A, Inoue A. Size dependence of soft to hard magnetic transition. Materialis Science and Engineering: A. 2004;375377:1140-1144. doi:10.1016/j.msea.2003.10.201

50. Wang YT, Xi XK, Fang YK, Zhao DQ, Pan MX, Han BS, et al. $\mathrm{Tb}$ nanocrystalline array assembled directly from alloy melt. Applied Physics Letters. 2004; 85(24):5989-5991.

51. Guo FQ, Poon SJ, Shiflet GJ. Metallic glass ingots based on yttrium. Applied Physics Letters. 2003;83(13):2575-2577.

52. Wang J, Li R, Hua N, Huang L, Zhang T. Ternary Fe-P-C bulk metallic glass with good soft-magnetic and mechanical properties. Scripta Materialia. 2011;65(6):536-539.

53. Jiang QK, Zhang GQ, Yang L, Wang XD, Saks K, Franz H, et al. La-based bulk metallic glasses with critical diameter up to 30 mm. Acta Materialia. 2007;55(13):4409-4418. DOI: 10.1016/j. actamat.2007.04.021

54. Schroers J, Johnson WL. Highly processable bulk metallic glass-forming alloys in the Pt-Co-Ni-Cu-P system. Applied Physics letters. 2004;84(18):3666-3668.

55. Zhang C, Li N, Pan J, Guo SF, Zhang M, Liu L. Enhancement of glass-forming ability and bio-corrosion resistance of Zr-Co-Al bulk metallic glasses by the addition of Ag. Journal of Alloys and Compounds. 2010;504S:S163-S167.

56. Xia L, Chan KC, Tang MB. Enhanced glass forming ability and refrigerant capacity of a Gd55Ni22Mn3 Al20 bulk metallic glass. Journal of Alloys and Compounds. 2011;509(23): 66406643. DOI: 10.1016/j.jallcom.2011.03.120

57. Kawashima A, Zeng Y, Fukuhara M, Kurishita H, Nishiyama $\mathrm{N}$, Miki H, et al. Mechanical properties of a Ni60Pd20P17B3 bulk glassy alloy at cryogenic temperatures. Materials Science and Engineering A. 2008;498(1-2):475-481. DOI:10.1016/j. msea.2008.08.033

58. Zhang L, Cheng YQ, Cao AJ, Xu J, Ma E. Bulk metallic glasses with large plasticity: Composition design from the structural perspective. Acta Materialia. 2009;57(4):1154-1164.

59. Huang XM, Chang CT, Chang ZY, Inoue A, Jiang JZ. Glass forming ability, mechanical and magnetic properties in $\mathrm{Fe}-\mathrm{W}$ Y-B alloys. Materials Science and Engineering A. 2010;527(78):1952-1956. DOI: 10.1016/j.msea.2009.11.042

60. Jiang QK, Wang XD, Nie XP, Zhang GQ, Ma H, Fecht $\mathrm{HJ}$, et al. $\mathrm{Zr}-(\mathrm{Cu}, \mathrm{Ag})-\mathrm{Al}$ bulk metallic glasses. Acta Materialia. 2008;56(8):1785-1796.

61. Angell CA. Structural instability and relaxation in liquid and glassy phases near the fragile liquid limit. Journal of NonCrystalline Solids. 1988;102(1-3):205-221.doi:10.1016/00223093(88)90133-0

62. Angell CA. Relaxation in liquids, polymers and plastic crystals strong/fragile patterns and problems. Journal of Non-Crystalline Solids. 1991;131-133(Part 1):13-31.

63. Lafi OA, Imran MM. Compositional dependence of thermal stability, glass-forming ability and fragility index in some Se-Te-Sn glasses. Journal of Alloys and Compounds. 2011;509(16):50905094. 